\title{
Relevance of Syntax Score as a Marker of Complex Coronary Artery Disease and Plaque Burden in Young Coronary Artery Disease - A Single Centre Multifactorial Analysis
}

\author{
Vinodkumar Balakrishnan ${ }^{1}$, Ramesh Sankaran², Thanikachalam S. ${ }^{3}$, Muralidharan T. R. ${ }^{4}$, \\ Nagendra Boopathy Senguttuvan ${ }^{5}$, Bhavidra Ramkumar 6
}

${ }^{1}$ Assistant Professor, Department of Cardiology, Sri Ramachandra Institute of Higher Education and Research (Deemed to be University), Chennai, Tamilnadu, India. ${ }^{2}$ Associate Professor, Department of Cardiology, Sri Ramachandra Institute of Higher Education and Research (Deemed to be University), Chennai, Tamilnadu, India. ${ }^{3}$ Professor, Department of Cardiology, Sri Ramachandra Institute of Higher Education and Research (Deemed to be University), Chennai, Tamilnadu, India. ${ }^{4}$ Professor, Department of Cardiology, Sri Ramachandra Institute of Higher Education and Research (Deemed to be University), Chennai, Tamilnadu, India. ${ }^{5}$ Assistant Professor, Department of

Cardiology, Sri Ramachandra Institute of Higher Education and Research (Deemed to be University), Chennai, Tamilnadu, India. ${ }^{6}$ Resident, Department of Cardiology, Sri Ramachandra Institute of Higher Education and Research

(Deemed to be University), Chennai, Tamilnadu, India.

\section{ABSTRACT}

\section{BACKGROUND}

Coronary Artery Disease (CAD) occurring in $<45$ years of age is termed as young CAD. The prevalence of CAD in this age group among Indians is $5-10 \%$, which is higher than the western average of $1.2 \%$. Smoking is the most critical risk factor in young CAD. Two factors associated with adverse prognosis are current smoking and obesity. Angiographic studies have shown predominantly single-vessel disease and lesser atheroma burden.

\section{METHODS}

It is a retrospective study conducted among young CAD adults aged between 20-45 years admitted for the acute coronary syndrome (ACS). We wanted to analyse the severity and extent of CAD by correlating with SYNTAX score and prevalence of conventional cardiovascular risk factors among those young Coronary Artery Disease patients.

\section{RESULTS}

In this study, 100 patients ( $83 \%$ male and $17 \%$ female), with a mean age of 39.9 years were included. Diabetes mellitus, hypertension, dyslipidaemia, hypothyroidism and history of young CAD and myocardial infarction were all positively related with a higher SYNTAX score (18\%) but were statistically insignificant due to relatively small sample size. Ejection fraction was lower in patients with higher syntax score $(>20 \%)$.

\section{CONCLUSIONS}

In young CAD undergoing coronary angiography, non-modifiable cardiovascular risk factors such as male sex, as well as other risk factors like hypertension, hypothyroidism, and dyslipidaemia, were independently associated with CAD complexity as assessed by SYNTAX score. SYNTAX score may be a valid marker for CAD to establish relationships with potential risk factors of coronary atherosclerosis.

\section{KEY WORDS}

SYNTAX Score, Hypertension, Type 2 Diabetes Mellitus, Hypothyroidism, Dyslipidemia, Coronary Angiography, Coronary Artery Disease
Corresponding Author:

Ramesh Sankaran,

Associate Professor,

Department of Cardiology,

Sri Ramachandra Institute of Higher

Education and Research

(Deemed to be University),

Chennai, Tamilnadu, India.

E-mail: sankaran_ramesh2000@yahoo.co.in

DOI: $10.14260 / \mathrm{jemds} / 2019 / 660$

Financial or Other Competing Interests: None.

How to Cite This Article:

Balakrishnan $V, \quad$ Sankaran $R$,

Thanikachalam $S$, et al. Relevance of syntax score as a marker of complex coronary artery disease and plaque burden in young coronary artery disease- a single centre multifactorial analysis. J. Evolution Med. Dent. Sci. 2019;8(40):3039-3043, DOI: 10.14260/jemds/2019/660

Submission 21-06-2019,

Peer Review 18-09-2019,

Acceptance 25-09-2019,

Published 07-10-2019. 


\section{BACKGROUND}

Acute Coronary Syndrome (ACS) has increased morbidity and mortality in developed countries in recent years with growing health care costs. Whereas, in developed and developing countries, the leading cause of morbidity and mortality is Coronary artery disease (CAD). World Health Organisation reported that burden due to CAD is increasing globally from 47 million to 82 million in 2020. Among which young CAD contributes to $2-6 \%$ of all ACS.[1] Recently, one of the significant issue cardiologists addressing in risk stratification in patients with ACS was to study the severity and complexity of CAD. ${ }^{[2]}$ A large number of scoring systems and laboratory parameters are in clinical practice to assess the severity and complexity of CAD. The most commonly used method is the SYNTAX.

SYNTAX is a significant scoring system predicting the prognosis and the need for revascularization. Also, it can determine the extent and severity of CAD. [3] The SYNergy between the percutaneous coronary intervention (PCI) with Taxus and cardiac surgery (SYNTAX) score calculated the complexity of CAD based on invasive coronary angiography (ICA).[3] The SYNTAX score can predict major adverse cardiac and cerebrovascular events (MACCEs) in populations with complex CAD (LM disease or 3-vessel disease).[4] It guides the optimal revascularization strategy. Among patients with complex CAD, Coronary artery By-pass grafting (CABG) remains as the standard of care for patients with high $(\geq 33)$ or intermediate (23-32) SYNTAX scores, whereas PCI is an acceptable alternative in patients with the less complicated disease (SYNTAX score 22). ${ }^{[5]}$ Although the scoring system has a large number of advantages, it requires an invasive method such as coronary angiography for performing the scoring. The Coronary Angiogram findings predict cardiac events and mortality in patients with CAD.[6,7] In this retrospective study on young CAD adults of age between 20-45 years with firsttime myocardial infarction, we analysed the severity and extent of CAD with syntax score and prevalence of conventional risk factors among those young CAD. The primary objective is to determine the severity of CAD among young CAD by correlating with syntax score. Other goals were to characterize the presence of cardiovascular risk factor among young patients admitted with myocardial infarction (MI) and to describe the coronary angiogram findings among young individuals with MI.

\section{METHODS}

Patient Data collected from Sri Ramachandran Medical University database. The patients of both sex with age $<45$ years, admitted with the acute coronary syndrome and have undergone coronary angiogram from January 2018 to January 2019 included in the study. Patients with raise or fall of cardiac biomarker $>1$ value above the upper reference range, symptoms suggestive of ischemia, significant ST-T wave changes / LBBB, pathologic Q in ECG, image evidence of loss of viable or new RWMA and intracoronary thrombus by CAG included. The patients with known CAD, before MI/ PCI / CABG, severe renal dysfunction, myositis, acute PTE, stroke excluded from the study. Demographic and cardiovascular risk factors data collected from the medical records.

Hypertension is said to be present when systolic blood pressure was $\geq 140 \mathrm{mmHg}$ and/or diastolic blood pressure $\geq 90$ mmHg or if patients is on antihypertensive medications. Diabetes considered present if the subject if fasting blood glucose was $\geq 110 \mathrm{mg}$ or postprandial blood glucose $\geq 140 \mathrm{mg}$ at admission or treated with insulin or oral hypoglycaemic drugs. Dyslipidaemia defined as total cholesterol $>200 \mathrm{mg} / \mathrm{dl}$, low-density lipoprotein (LDL) cholesterol $>130 \mathrm{mg} / \mathrm{dl}$ or on lipid-lowering medications.

\section{Coronary Angiography}

Details of coronary angiogram were obtained from cardiac catheterisation laboratory records and were evaluated. Coronary angiography (CAG) was performed on all our patients by either femoral or radial approach. A $6 \mathrm{~F}$ diagnostic catheter and iopromide as a contrast agent (Ultravist-370, Bayer Schering Pharma, Germany) was used in all subjects. A diameter of stenosis calculated as $\geq 50 \%$ with quantitative angiography accepted as significant. SYNTAX score was used to define the coronary atherosclerotic lesion. SYNTAX score calculated by two of three experienced cardiologists blinded in the study. If there was any controversy, the opinion of the third cardiologist was asked, and the last conclusion made with consensus. All coronary lesion with $50 \%$ diameter stenosis or more in arteries of at least $1.5 \mathrm{~mm}$ were scored. To assess the SYNTAX score most recently updated version used for the calculation (http://www.SYNTAXscore.com). SYNTAX score > 20 defined as high SYNTAX scores.

\section{Statistical Analysis}

Statistical analyses performed by SPSS (version 15.0, SPSS, Chicago, Illinois, USA). Quantitative variables expressed as the mean \pm standard deviation. Between-group differences of the categorical variable were analysed using the Chi-square test and the Student t-test for continuous variables. P-value $<0.05$ was considered statistically significant.

\section{RESULTS}

This retrospective study included data of 100 patients diagnosed with ACS and aged between 20 years to 45 years. Demographic data revealed ACS prevalence of $51 \%, 48 \%$ and $01 \%$ in patients between age group $31-40,40-45$, and 20-30, respectively. There was male preponderance in the prevalence of ACS with $83 \%$ in this study. Most of the study subjects $(82 \%)$ had a SYNTAX score of $\leq 20$ (Syn $\leq 20$ ) and $18 \%$ exhibited SYNTAX score of $>20$ (Syn $>20$ ) [Table 1].

\section{CAG SYNTAX Score and Prevalence of Risk Factors}

SYNTAX scores of ACS patients obtained from CAG findings categorized as Syn $\leq 20$ and Syn $>20$. The Syn $\leq 20$ seen in 82 patients, which included 67 males and 15 females with a mean age of 39.58 years. The Syn $>20$ seen in 18 patients, which included 16 males and two females with a mean age of 41.33 years. The gender distribution, mean age range between the two categories of SYNTAX scores were comparable and statistically insignificant the prevalence of risk factors associated between the two categories of SYNTAX scores 
evaluated with the chi-square test. Prevalence of T2DM seen in 22 patients with Syn $\leq 20$ and five patients with Syn $>20$. History of dyslipidaemia seen in 10 patients with Syn $\leq 20$ and six patients with Syn $>20$. Hypothyroidism reported by three patients with Syn $\leq 20$. Hypertension diagnosed in 10 patients with Syn $<20$ and 5 patients with Syn $>20$. Totally four patients were smoking history, and all had syntax score $\leq 20$. Mean left ventricle ejection fraction was seen decreased in Syn $>20$ when compared to Syn $\leq 20$, but statistically insignificant (p-value $=0.07)$ [Table 2].

\begin{tabular}{|c|c|c|c|}
\hline Sl. No. & Characteristics & Frequency (n) & Percentage (\%) \\
\hline \multirow{3}{*}{1} & \multicolumn{3}{|c|}{ SYNTAX Score } \\
\cline { 2 - 4 } & $\leq 20$ & 82 & 82 \\
\cline { 2 - 4 } & $>20$ & 18 & 18 \\
\hline \multirow{3}{*}{2} & \multicolumn{3}{|c|}{ Sex } \\
\cline { 2 - 4 } & Male & 83 & 83 \\
\cline { 2 - 4 } & Female & 17 & 17 \\
\hline \multirow{3}{*}{3} & \multicolumn{3}{|c|}{ Age } \\
\cline { 2 - 4 } & $20-30$ & 51 & 51 \\
\cline { 2 - 4 } & $31-40$ & 48 & 48 \\
\cline { 2 - 4 } & $41-45$ & \multicolumn{3}{|c|}{ Table 1. Patient Characteristics of the Study Group } \\
\hline \multicolumn{3}{|c|}{}
\end{tabular}

\begin{tabular}{|c|c|c|c|c|}
\hline Sl. No. & Risk Factors & SYNTAX Score $\leq \mathbf{2 0}$ & SYNTAX Score $>\mathbf{2 0}$ & p \\
\hline 1 & Male (n) & 67 & 16 & 0.825 \\
\hline 2 & Female (n) & 15 & 2 & 0.527 \\
\hline 3 & Age (Mean \pm SD) & $39.58 \pm 4.21$ & $41.33 \pm 3.08$ & 0.482 \\
\hline 4 & T2DM (n) & 22 & 5 & 0.984 \\
\hline 5 & Hypothyroidism (n) & 3 & 0 & 0.418 \\
\hline 6 & HTN (n) & 10 & 5 & 0.166 \\
\hline 7 & DLP (n) & 10 & 6 & .0738 \\
\hline 8 & LVEF (\%) (Mean \pm SD) & $54.87 \pm 9.84$ & $49.64 \pm 13.56$ & 0.075 \\
\hline 9 & Smoking & 4 & 0 & 0.491 \\
\hline \multicolumn{5}{|c|}{ Table 2. SYNTAX Scores and Prevalence of Risk Factors } \\
\hline \multicolumn{5}{|c}{} \\
\hline
\end{tabular}

\section{DISCUSSION}

Prevalence of Coronary artery disease over the last few decades increased in young age (Below 45 years) due to increase in risk factors in the populations. The prognosis as well as longevity after an acute coronary episode in this age group is varied compared to conventional age. In this study, SYNTAX score was calculated for all patients and the severity of CAD were measured with SYNTAX scores. This study evaluated the relationship between SYNTAX score obtained from CAG and conventional CV risk factors, in young CAD diagnosed with acute coronary syndrome. This study demonstrated a positive correlation of SYNTAX score with age, diabetes mellitus, dyslipidaemia, hypertension, and hypothyroidism. In our study persons with syntax score more than 20 had lower ejection fraction by echocardiogram compared to persons syntax score less than 20. SYNTAX score has become an reliable method to evaluate CAD complexity. ${ }^{[8,9,10]}$ SYNTAX score was chosen for this study, due to its strict anatomical based design to assess CAD complexity.[1] Despite its limitations[11] and criticism in current clinical practice, SYNTAX trial still applies.[12] SYNTAX score used as a surrogate marker which sought to establish correlations of several clinical and biochemical variables with coronary atherosclerosis.[13,14,15] However, the SYNTAX score in this context has not validated correctly. Framingham Heart study and many landmark studies has established the role of age, male gender, diabetes mellitus, hypertension, hypothyroidism, and lipid abnormalities as risk factors for CAD. $[16,17,18]$ This study suggests a positive association of these parameters with the complexity of $\mathrm{CAD}$, as expressed through
SYNTAX score. Male preponderance (83\%) might be associated with progressive endothelial dysfunction, occurring earlier in males, presumably due to the protective role of oestrogens in premenopausal women. ${ }^{(19,20)}$ In diabetes hyperglycaemia, insulin resistance and free fatty acid release have been shown to lead to increased oxidative stress and therefore accelerate atherosclerosis.(21,22,23,24,25) In the present study, 22 of 82 patients had a history of T2DM with SYN $\leq 20$, and five of 18 patients had a history of T2DM with SYN $>20$. The score should be applied for major epicardial coronaries, including mainly coronary lesions located in vessels with diameters $>1.5 \mathrm{~mm}$. Therefore, CAD extensiveness in diabetic patients underestimated with this method. Due to prevention strategies, the well-known vascular risk factors like dyslipidaemia or hypertension not associated with the score. Lack of correlation is mainly due to prior treatment with statins to reduce the concentration of LDL-c, triglyceride, Apo$B$, increase in HDL-c and treatment with angiotensin-II receptor blocking agents to lower the rate of plaque burden and coronary atheroma progression.(26,27) As mentioned above, the SYNTAX score is used as a surrogate marker of CAD. Variables like fasting blood glucose, monocyte subtypes, red cell distribution width or bilirubin levels were compared with SYNTAX score in the development of coronary atherosclerosis. $(28,29,30,31,32)$ We believe that the demonstrated relationship of 'non-modifiable' risk factors with the complexity of CAD, justifies the use of SYNTAX score in assessing the severity of coronary artery disease in young patients.

\section{CONCLUSIONS}

In young CAD undergoing coronary angiography, nonmodifiable cardiovascular risk factors such as male sex, as well as other risk factors like hypertension, hypothyroidism, and dyslipidaemia, were independently associated with CAD complexity as assessed by SYNTAX score. SYNTAX score seems to be a valid marker of CAD to establish relationships with potential risk factors of coronary atherosclerosis.

\section{REFERENCES}

[1] Authors/Task Force members, Windecker S, Kolh P, et al. 2014 ESC/EACTS guidelines on myocardial revascularization: the Task Force on Myocardial Revascularization of the European Society of Cardiology (ESC) and the European Association for Cardio-Thoracic Surgery (EACTS) developed with the special contribution of the European Association of Percutaneous Cardiovascular Interventions (EAPCI). European Heart Journal 2014;35(37):2541-619.

[2] Arbel Y, Zlotnik M, Halkin A, et al. Admission glucose, fasting glucose, HbA1c levels and the SYNTAX score in non-diabetic patients undergoing coronary angiography. Clinical Research in Cardiology 2014;103(3):223-7.

[3] Sianos G, Morel MA, Kappetein AP, et al. The SYNTAX Score: an angiographic tool grading the complexity of coronary artery disease. EuroIntervention 2005;1(2):219-27. 
[4] Sinning C, Lillpopp L, Appelbaum S, et al. Angiographic score assessment improves cardiovascular risk prediction: the clinical value of SYNTAX and Gensini application. Clinical Research in Cardiology 2013;102(7):495-503.

[5] Mohr FW, Morice MC, Kappetein AP, et al. Coronary artery bypass graft surgery versus percutaneous coronary intervention in patients with three-vessel disease and left main coronary disease: 5-year follow-up of the randomised, clinical SYNTAX trial. The Lancet 2013;381(9867):629-38.

[6] Das MK, Michael MA, Suradi $H$, et al. Usefulness of fragmented QRS on a 12-lead electrocardiogram in acute coronary syndrome for predicting mortality. The American Journal of Cardiology 2009;104(12):1631-7.

[7] Das MK, Saha C, El Masry H, et al. Fragmented QRS on a 12-lead ECG: a predictor of mortality and cardiac events in patients with coronary artery disease. Heart Rhythm 2007;4(11):1385-92.

[8] Farooq V, Van Klaveren D, Steyerberg EW, et al. Anatomical and clinical characteristics to guide decision making between coronary artery bypass surgery and percutaneous coronary intervention for individual patients: development and validation of SYNTAX score II. The Lancet 2013;381(9867):639-50.

[9] Serruys PW, Morice MC, Kappetein AP, et al. Percutaneous coronary intervention versus coronary-artery bypass grafting for severe coronary artery disease. New England Journal of Medicine 2009;360(10):961-72.

[10] Head SJ, Davierwala PM, Serruys PW, et al. Coronary artery bypass grafting vs. percutaneous coronary intervention for patients with three-vessel disease: final five-year follow-up of the SYNTAX trial. European Heart Journal 2014;35(40):2821-30.

[11] Capodanno D, Tamburino C. Does the SYNTAX score get on your nerves? Practical considerations on how and when avoiding it to maximize its usefulness with no waste of time. Int J Cardiol 2012;159(3):165-8.

[12] Claessen BE, Stone GW, Smits PC, et al. Would SYNTAX have been a positive trial if XIENCE $\mathrm{V}$ had been used instead of TAXUS? A meta-analysis of a first-generation vs. a second-generation drug-eluting stent system. Netherlands Heart Journal 2010;18(9):451-3.

[13] Gijsberts CM, Seneviratna A, Bank IE, et al. The ethnicityspecific association of biomarkers with the angiographic severity of coronary artery disease. Netherlands Heart Journal 2016;24(3):188-98.

[14] Yuksel IO, Cagirci G, Koklu E, et al. Erythropoietin stimulates the coronary collateral development in patients with coronary chronic total occlusion. Netherlands Heart Journal 2016;24(10):609-16.

[15] Tanaka T, Seto S, Yamamoto K, et al. An assessment of risk factors for the complexity of coronary artery disease using the SYNTAX score. Cardiovascular Intervention and Therapeutics 2013;28(1):16-21.

[16] Keys A. Coronary heart disease in seven countries: summary. Circulation 1970;41(1):186-95

[17] Labounty TM, Gomez MJ, Achenbach S, et al. Body mass index and the prevalence, severity and risk of coronary artery disease: an international multicentre study of 13 874 patients. European Heart Journal-Cardiovascular Imaging 2013;14(5):456-63.
[18] Mahmood SS, Levy D, Vasan RS, et al. The Framingham Heart Study and the epidemiology of cardiovascular disease: a historical perspective. The Lancet 2014;383(9921):999-1008.

[19] Celermajer DS, Sorensen KE, Spiegelhalter DJ, et al. Aging is associated with endothelial dysfunction in healthy men years before the age-related decline in women. Journal of the American College of Cardiology 1994;24(2):471-6.

[20] Cepeda-Valery B, Chaudhry K, Slipczuk L, et al. Association between obesity and severity of coronary artery disease at the time of acute myocardial infarction: another piece of the puzzle in the "obesity paradox". International Journal of Cardiology 2014;176(1):247-9.

[21] Creager MA, Lüscher TF, Cosentino F, et al. Diabetes and vascular disease: pathophysiology, clinical consequences and medical therapy: Part I. Circulation 2003;108(12):1527-32.

[22] Das MK, Suradi H, Maskoun W, et al. Fragmented wide QRS on a 12-lead ECG: a sign of myocardial scar and poor prognosis. Circulation: Arrhythmia and Electrophysiology 2008;1(4):258-68.

[23] Dores H, de Araújo GP, Carvalho MS, et al. Body mass index as a predictor of the presence but not the severity of coronary artery disease evaluated by cardiac computed tomography. European Journal of Preventive Cardiology 2014;21(11):1387-93.

[24] Fihn SD, Blankenship JC, Alexander KP, et al. 2014 ACC/AHA/AATS/PCNA/SCAI/STS focused update of the guideline for the diagnosis and management of patients with stable ischemic heart disease: a report of the American College of Cardiology/American Heart Association Task Force on Practice Guidelines, and the American Association for Thoracic Surgery, Preventive Cardiovascular Nurses Association, Society for Cardiovascular Angiography and Interventions, and Society of Thoracic Surgeons. Journal of the American College of Cardiology 2014;64(18):1929-49.

[25] Gensini GG. A more meaningful scoring system for determining the severity of coronary heart disease. Am J Cardiol 1983;51(3):606.

[26] Hirohata A, Yamamoto K, Miyoshi T, et al. Impact of olmesartan on progression of coronary atherosclerosis: a serial volumetric intravascular ultrasound analysis from the OLIVUS (impact of olmesarten on progression of coronary atherosclerosis: evaluation by intravascular ultrasound) trial. Journal of the American College of Cardiology 2010;55(10):976-82.

[27] Jukema JW, Bruschke AV, van Boven AJ, et al. Effects of lipid lowering by pravastatin on progression and regression of coronary artery disease in symptomatic men with normal to moderately elevated serum cholesterol levels: the Regression Growth Evaluation Statin Study (REGRESS). Circulation 1995;91(10):252840.

[28] Kalay N, Dogdu O, Koc F, et al. Hematologic parameters and angiographic progression of coronary atherosclerosis. Angiology 2012;63(3):213-7.

[29] Korhonen P, Husa T, Konttila T, et al. Fragmented QRS in prediction of cardiac deaths and heart failure hospitalizations after myocardial infarction. Annals of Noninvasive Electrocardiology 2010;15(2):130-7. 
[30] Pietrasik G, Goldenberg I, Zdzienicka J, et al. Prognostic significance of fragmented QRS complex for predicting the risk of recurrent cardiac events in patients with Qwave myocardial infarction. The American Journal of Cardiology 2007;100(4):583-6.

[31] Romero-Corral A, Montori VM, Somers VK, et al. Association of bodyweight with total mortality and with cardiovascular events in coronary artery disease: a systematic review of cohort studies. The Lancet 2006;368(9536):666-78.
[32] Turfan M, Duran M, Poyraz F, et al. Inverse relationship between serum total bilirubin levels and severity of disease in patients with stable coronary artery disease. Coronary Artery Disease 2013;24(1):29-32. 